\title{
Monitoring and interpretation of intracranial pressure
}

\author{
M Czosnyka, J D Pickard
}

J Neurol Neurosurg Psychiatry 2004;75:813-821. doi: 10.1136/jnnp.2003.033126

Intracranial pressure (ICP) is derived from cerebral blood and cerebrospinal fluid (CSF) circulatory dynamics and can be affected in the course of many diseases of the central nervous system. Monitoring of ICP requires an invasive transducer, although some attempts have been made to measure it non-invasively. Because of its dynamic nature, instant CSF pressure measurement using the height of a fluid column via lumbar puncture may be misleading. An averaging over 30 minutes should be the minimum, with a period of overnight monitoring in conscious patients providing the optimal standard. Computer-aided recording with online waveform analysis of ICP is very helpful. Although there is no "Class I" evidence, ICP monitoring is useful, if not essential, in head injury, poor grade subarachnoid haemorrhage, stroke, intracerebral haematoma, meningitis, acute liver failure, hydrocephalus, benign intracranial hypertension, craniosynostosis etc. Information which can be derived from ICP and its waveforms includes cerebral perfusion pressure (CPP), regulation of cerebral blood flow and volume, CSF absorption capacity, brain compensatory reserve, and content of vasogenic events. Some of these parameters allow prediction of prognosis of survival following head injury and optimisation of "CPP-guided therapy". In hydrocephalus CSF dynamic tests aid diagnosis and subsequent monitoring of shunt function.

See end of article for authors' affiliations

Correspondence to: DrM Czosnyka, Academic Neurosurgery, Box 167 Addenbrooke's Hospital Cambridge CB2 2QQ, UKK; MC141@medschl.cam.ac. uk

Received

21 November 2003

In revised form

20 January 2004

Accepted 22 January 2004 $\mathrm{n}$ most organs of the human body the environmental pressure for blood perfusion is either low, or coupled to atmospheric pressure. The environmental pressure for the brain differs in this respect as brain is surrounded and protected by a stiff skull. A rise in intracranial pressure (ICP) may impede blood flow and cause ischaemia.

Intracranial pressure is derived from the circulation of cerebral blood and cerebrospinal fluid (CSF) (however, it is not certain whether the operator in the formula should be represented by a simple addition): ICP $=\mathrm{ICP}_{\text {vascular }}+$ ICP $_{\text {CSF }}$.

The vascular component ${ }^{12}$ is difficult to express quantitatively. It is probably derived from the pulsation of the cerebral blood volume detected and averaged by non-linear mechanisms of regulation of cerebral blood volume. More generally, multiple variables such as the arterial pressure, autoregulation, and cerebral venous outflow all contribute to the vascular component. The other circulatory CSF component may be expressed using Davson's equation ${ }^{3}$ : $\mathrm{ICP}_{\mathrm{CSF}}=($ resistance to CSF outflow $) \times(\mathrm{CSF}$ formation $)+$ (pressure in sagittal sinus).

Any factor, which under physiological (for example, compression of jugular veins during a Queckenstedt test) or pathological conditions (brain swelling, space occupying lesion, obstruction of CSF pathway) disturbs this circulation, may provoke an increase in ICP.

ICP measurements are used to estimate cerebral perfusion pressure (CPP) as follows: mean $\mathrm{CPP}=$ mean arterial blood pressure (ABP) - mean ICP. CPP represents the pressure gradient acting across the cerebrovascular bed, and hence is an important factor in regulation of the cerebral blood flow (CBF). ${ }^{4}$ Sufficient CPP is required to maintain a stable $\mathrm{CBF}$. The autoregulatory reserve is interpreted as the difference between current mean CPP and lower limit of autoregulation. Low CPP (a threshold of 60$70 \mathrm{~mm} \mathrm{Hg}$ is generally accepted in adults) may result in exhaustion of the autoregulatory reserve. However, policies to therapeutically maintain a high CPP are controversial. If the cerebral vessels are non-reactive, an increase in CPP may result in hyperaemia, increase in vasogenic oedema, and a secondary increase in ICP. ${ }^{56}$ It is also probable that patient and time dependent differences in the optimal level of CPP may be considerable. Thus, the border between adequate and non-adequate CPP should be assessed individually and frequently.

\section{METHODS OF MEASUREMENT}

An intraventricular drain connected to an external pressure transducer is still considered to be "golden standard" method. ${ }^{78}$ ICP can be controlled by CSF drainage and the transducer may be zeroed externally. However, after five days of monitoring the risk of infection starts to increase, with an overall risk estimated to be about 5\%.' Insertion of the ventricular catheter may be difficult or impossible in cases of advanced brain swelling. Modern ventricular, subdural, or intraparenchymal microtransducers (most popular types: Camino ICP Bolt; Camino Laboratories, San Diego, California, USA; and Codman MicroSensor, Johnson and Johnson Professional Inc, Raynham, Massachusetts, USA) reduce infection rate and risk of haemorrhage ${ }^{9}$ and have excellent metrological properties as revealed during bench tests- that is, bandwidth,

Abbreviations: $A B P$, arterial blood pressure; $A M P$, pulse amplitude of ICP; CBF, cerebral blood flow; CPP, cerebral perfusion pressure; CSF, cerebrospinal fluid; ICP intracranial pressure; $P R x$, pressure-reactivity index; RAP, index of compensatory reserve; Slow, magnitude of slow waves of ICP 
linearity. ${ }^{10}$ Generally, uniformly distributed ICP can be probably seen only when CSF circulates freely between all its natural pools, equilibrating pressure everywhere. When little or no CSF volume is left due to brain swelling, the assumption of one, uniform value of ICP is questionable. With the most common intraparenchymal probes, measured pressure may be compartmentalised and not necessarily representative of real ICP - that is, ventricular CSF pressure. ${ }^{11}$ Microtransducers cannot be generally re-zeroed after insertion and considerable zero drift can sometimes occur in long term monitoring. ${ }^{12}$ This problem has been addressed in the balloon-like Spiegelberg transducer which may zero itself every set time interval, although its limited bandwidth may make most of the methods used for the ICP waveform analysis impossible. ${ }^{13}$ Contemporary epidural sensors are much more reliable than 10 years ago. But the question as to whether epidural pressure can express ICP with confidence and under all circumstances is still unanswered.

Lumbar CSF pressure is very seldom measured in neurointensive care. This form of assessment of craniospinal dynamics is more often used in hydrocephalus and benign intracranial hypertension. It is important to emphasise that decent monitoring over a period of at least half an hour with recording of pressure and pulse amplitude should be used as a "golden standard". Manometric assessment by measuring the height of the CSF column may be misleading as CSF pressure may vary considerably with time. ${ }^{14}$ Attempts to monitor ICP non-invasively are still in a phase of technical evaluation. ${ }^{15}$ The most promising methods are based on transcranial ultrasonography $y^{16-18}$ - see below.

\section{TYPICAL EVENTS AND TRENDS IN ICP MONITORING}

It is difficult to establish a universal "normal value" for ICP as it depends on age, body posture, and clinical conditions. In the horizontal position, the normal ICP in healthy adult subjects was reported to be within the range of 7-15 mm $\mathrm{Hg} .{ }^{19}$ In the vertical position it is negative with a mean of around $-10 \mathrm{~mm} \mathrm{Hg}$, but not exceeding $-15 \mathrm{~mm} \mathrm{Hg} .{ }^{20}$

The definition of raised ICP depends on the specific pathology. In hydrocephalus, a pressure above $15 \mathrm{~mm} \mathrm{Hg}$ can be regarded as elevated. Following head injury, anything above $20 \mathrm{~mm} \mathrm{Hg}$ is abnormal and aggressive treatment usually starts above $25 \mathrm{~mm} \mathrm{Hg}$. It is important to recognise that ICP in most cases varies with time. Decent averaging for at least 30 minutes is needed to calculate "mean ICP". The patient should rest in a horizontal position during the measurement and avoid movement, speaking, etc. Overnight monitoring during natural sleep, which provides a "grand average" with a good description of the dynamics of the pressure, should be regarded as the "gold standard" in conscious patients.

When monitored continuously in acute states (head injury, poor grade subarachnoid haemorrhage, intracerebral haematoma etc.), mean ICP may be classified into relatively few patterns (fig 1).

- Low and stable ICP (below $20 \mathrm{~mm} \mathrm{Hg}$ )-for example, seen in patients following uncomplicated head injury (fig 1A). Such a pattern is also commonly seen in the initial period after brain trauma before the brain swelling evolves.

- High and stable ICP (above $20 \mathrm{~mm} \mathrm{Hg}$ ) - the most common picture to follow head injury (fig 1B).

- Vasogenic waves- "B" waves (fig $1 \mathrm{C}$ ) and plateau waves (fig 1D).

- ICP waves related to changes in arterial pressure and hyperaemic events (fig l E-G).

- Refractory intracranial hypertension (fig $1 \mathrm{H}$ ). This usually leads to death unless radical measures-for example, surgical decompression, are applied.
However, there is much more information in the ICP waveform than in the time averaged ICP mean value alone. It is important to bear in mind how little information is available in the fluid column in the manometer line connected to a lumbar puncture needle!

\section{WAVEFORM ANALYSIS OF ICP}

The ICP waveform consists of three components, which overlap in the time domain, but can be separated in the frequency domain (fig 2). The pulse waveform has several harmonic components; of these the fundamental component has a frequency equal to the heart rate. The amplitude of this component (AMP) is very useful for the evaluation of various indices. The respiratory waveform is related to the frequency of the respiratory cycle (8-20 cycles per minute). "Slow waves" are usually not as precisely defined as in the original Lundberg thesis. ${ }^{8}$ All components that have a spectral representation within the frequency limits of $0.05-$ $0.0055 \mathrm{~Hz}$ ( $20 \mathrm{~s}$ to $3 \mathrm{~min}$ period) can be classified as slow waves. The magnitude of these waves can be calculated as the square root of the power of the signal of the passband of the equivalent frequency range at the output of the digital filter.

\section{PRESSURE-VOLUME COMPENSATORY RESERVE}

Theoretically, the compensatory reserve can be studied through the relation between ICP and changes in volume of the intracerebral space, known as the "pressure-volume curve". $22{ }^{23}$ The index called RAP (correlation coefficient (R) between AMP amplitude (A) and mean pressure (P); index of compensatory reserve) can be derived by calculating the linear correlation between consecutive, time averaged data points of AMP and ICP (usually 40 of such samples are used) acquired over a reasonably long period to average over respiratory and pulse waves (usually 6-10 s periods). This index indicates the degree of correlation between AMP and mean ICP over short periods of time $(\sim 4 \mathrm{~min})$.

A RAP coefficient close to 0 indicates lack of synchronisation between changes in AMP and mean ICP. This denotes a good pressure-volume compensatory reserve at low ICP (fig 3), where a change in volume produces no or very little change of the pressure. When RAP rises to +1 , AMP varies directly with ICP and this indicates that the "working point" of the intracranial space shifts to the right towards the steep part of the pressure-volume curve. Here compensatory reserve is low; therefore any further rise in volume may produce a rapid increase in ICP. Following head injury and subsequent brain swelling, RAP is usually close to +1 . With any further increase in ICP, AMP decreases and RAP values fall below zero. This occurs when the cerebral autoregulatory capacity is exhausted and the pressure-volume curve bends to the right as the capacity of cerebral arterioles to dilate in response to a CPP decrement is exhausted, and they tend to collapse passively. This indicates terminal cerebrovascular derangement with a decrease in pulse pressure transmission from the arterial bed to the intracranial compartment.

\section{CEREBROVASCULAR PRESSURE REACTIVITY}

Another ICP derived index is the pressure-reactivity index (PRx), which incorporates the philosophy of assessing cerebrovascular reactions by observing the response of ICP to slow spontaneous changes in $\mathrm{ABP} .^{24}$ When the cerebrovascular bed is normally reactive, any change in $A B P$ produces an inverse change in cerebral blood volume and hence ICP. When reactivity is disturbed, changes in ABP are passively transmitted to ICP. Using computational methods similar to those used for the calculation of the RAP index, PRx is determined by calculating the correlation coefficient between 40 consecutive, time averaged data points of ICP and ABP. A positive PRx signifies a positive gradient of the 

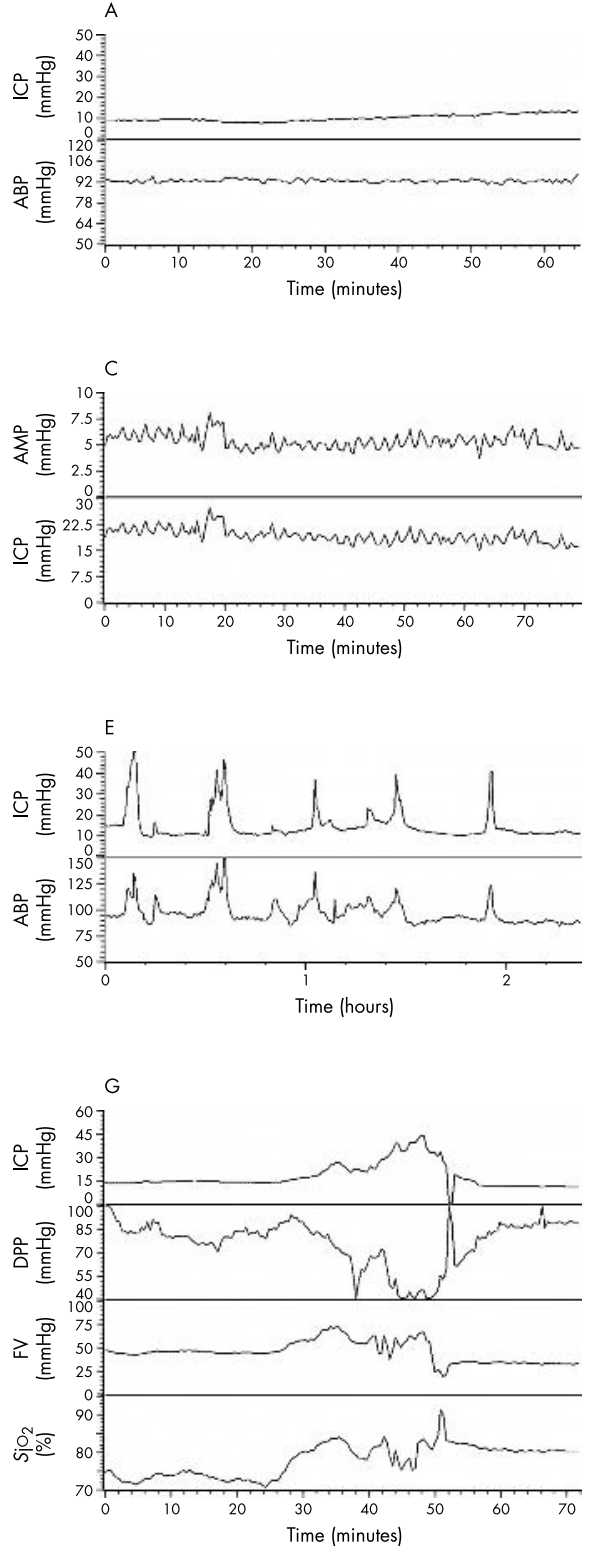

regression line between the slow components of $\mathrm{ABP}$ and ICP, which we hypothesise to be associated with passive behaviour of a non-reactive vascular bed (fig 4A). A negative value of PRx reflects a normally reactive vascular bed, as ABP waves provoke inversely correlated waves in ICP (fig 4B). This index correlates well with indices of autoregulation based on transcranial Doppler ultrasonography. ${ }^{25}$ Furthermore, abnormal values of both PRx and RAP, indicative respectively of poor autoregulation or deranged cerebrospinal compensatory reserve, have been demonstrated to be predictive of a poor outcome following head injury. ${ }^{5}$

\section{OTHER METHODS OF ICP ANALYSIS}

One of the priorities in brain monitoring is to develop a technique which helps in predicting decompensation or herniation. Early works focused on intracranial volumepressure response ${ }^{26}$ which evolved in time into continuous monitoring of brain compliance. ${ }^{27}$ Such a method relies on the evaluation of the pressure response to known small volume additions by inflating and deflating a balloon inserted within the cerebrospinal space. The method has been implemented in the Spiegelberg Brain Compliance
Figure 1 (A) Low and stable intracranial pressure (ICP). Mean arterial blood pressure (ABP) is plotted along the lower panel. (B) Stable and elevated ICP-this can be seen most of the time in head injury patients. (C) " $\mathrm{B}$ " waves of ICP. They are seen both in mean ICP and spectrally resolved pulse amplitude of ICP (AMP, upper panel). They are also usually seen in plots of time averaged $A B P$, but not always. (D) Plateau waves of ICP. Cerebrospinal compensatory reserve is usually low when these waves are recorded (RAP (correlation coefficient $(R)$ between AMP amplitude (A) and mean pressure (P)) close to +1 ; index of compensatory reserve). At the height of the waves, during maximal vasodilatation, integration between pulse amplitude and mean ICP fails as is indicated by fall in RAP. After the plateau wave, ICP usually falls below baseline level and cerebrospinal compensatory reserve improves. (E) High, spiky waves of ICP caused by sudden increases in ABP. (F) Increase in ICP caused by temporary decrease in ABP. (G) Increase in ICP of "hyperaemic nature". Both blood flow velocity and jugular venous oxygen saturation $\left(\mathrm{SjO}_{2--}\right)$ increased in parallel with ICP. (H) Refractory intracranial hypertension. ICP increased within a few hours to above $100 \mathrm{~mm} \mathrm{Hg}$. The vertical line denotes the moment when the ischaemic wave probably reached the vasomotor centres in the brain stem: heart rate increased and ABP (cerebral perfusion pressure) decreased abruptly. Note that the pulse amplitude of ICP (AMP) disappeared around 10 minutes before this terminal event. 

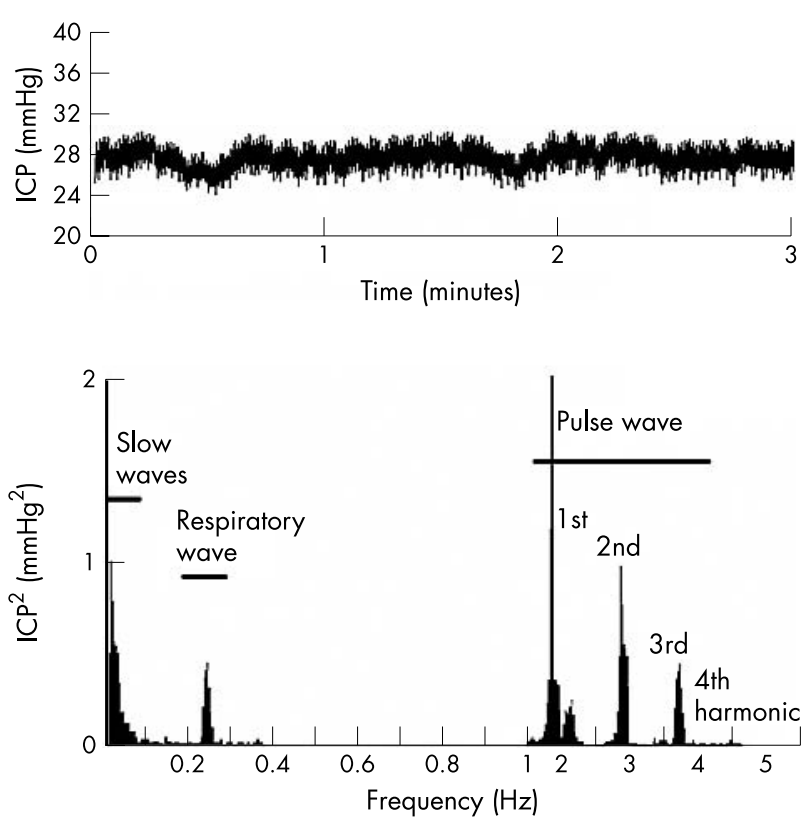

Figure 2 Example of ICP recording showing pulse, respiratory, and "slow waves" overlapped in time domain (upper panel). Taken from Balestreri M, Czosnyka M. Acta Neurochir 2004; (in press) ${ }^{21}$ with permission from the publishers.

Recently, the power of slow waves of ICP was reported to be predictive of outcome in patients with intracranial hypertension following head injury. A low content of slow waves in the overall ICP dynamics was associated with a fatal outcome $^{21}$ highlighting a possible link of these events with cerebral autoregulation. ${ }^{35}$

\section{PRACTICAL USE OF THE ICP DERIVED PARAMETERS: "OPTIMAL CPP" AND MULTIPLE TREND ANALYSIS}

Both PRx and RAP can be used to evaluate secondary variables that combine the value of absolute ICP and CPP with information about the state of autoregulatory and compensatory reserves. PRx plotted against CPP gives a " $U$ shape" curve. ${ }^{5}$ This indicates that for the majority of patients there is a value of the CPP in which pressure-reactivity is optimal. This optimal pressure can be estimated by plotting and analysing the PRx-CPP curve in a sequential six hour wide time-moving window online. It has been demonstrated in a group of retrospectively evaluated patients that the greater the distance between the current and the "optimal" CPP the worse the outcome. This potentially useful methodology attempts to refine CPP oriented therapy. ${ }^{5}$ Both, too low (ischaemia), and, too high, CPP (hyperaemia and secondary increase in ICP) are detrimental. Hence, we have suggested that CPP should be optimised to maintain cerebral perfusion in the globally most favourable state. ${ }^{5}$

No matter how sophisticated new variables or outcomepredicting models become, the most useful tool at the bedside is a computer screen, which presents the trends of multiple parameters with time. This gives an opportunity to react to a crisis situation, understand the cerebrospinal dynamics in multiple dimensions and predict an optimal strategy for the individual patient's care (fig 5).

\section{ASSOCIATION WITH OUTCOME FOLLOWING SEVERE HEAD INJURY}

In severe head injury an averaged ICP above $25 \mathrm{~mm} \mathrm{Hg}$ over the whole period of monitoring increases risk of death twofold. Averaged values of the RAP and PRx indices are also

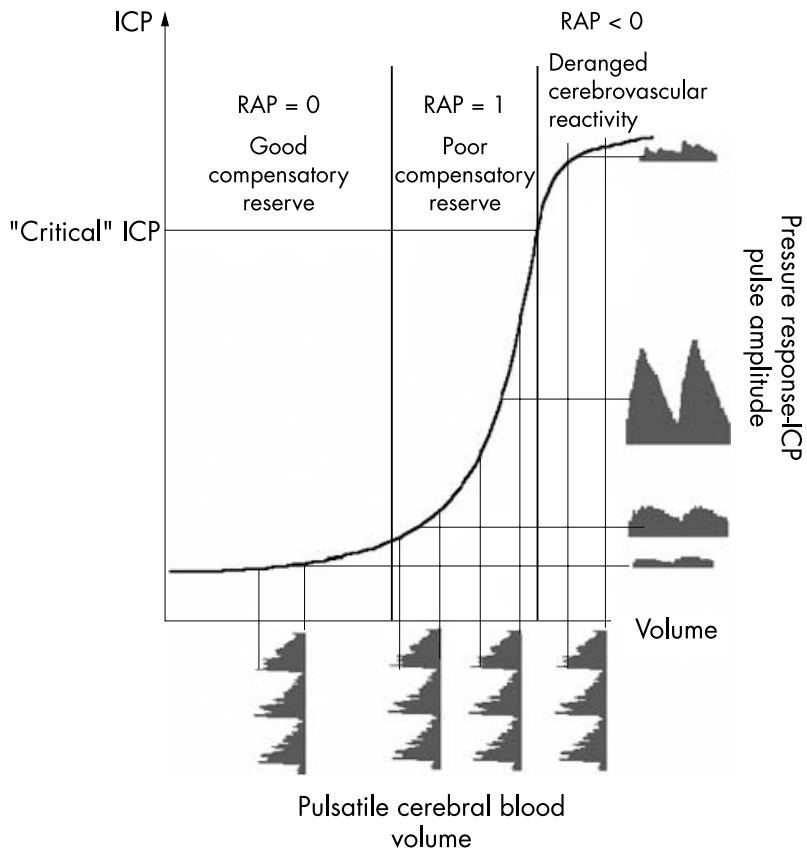

Figure 3 In a simple model, pulse amplitude of intracranial pressure (ICP) (expressed along the $y$-axis on the right side of the panel) results from pulsatile changes in cerebral blood volume (expressed along the $x$ axis) transformed by the pressure-volume curve. This curve has three zones: a flat zone, expressing good compensatory reserve, an exponential zone, depicting poor compensatory reserve, and a flat zone again, seen at very high ICP (above the "critical" ICP) depicting derangement of normal cerebrovascular responses. The pulse amplitude of ICP is low and does not depend on mean ICP in the first zone. The pulse amplitude increases linearly with mean ICP in the zone of poor compensatory reserve. In the third zone, the pulse amplitude starts to decrease with rising ICP. RAP, index of compensatory reserve. Taken from Balestreri M, Czosnyka M. Acta Neurochir 2004; (in press) ${ }^{21}$ with permission from the publishers.

strong predictors of fatal outcome. Both these indices suggest that good vascular reactivity is an important element of brain homoeostasis, enabling the brain to protect itself against an uncontrollable rise of the intracerebral volume. A low value of slow waves of ICP is also indicative of a fatal outcome following head injury.

As ICP, PRx, and the power of ICP slow waves are independent predictors of outcome, these three variables, although mutually correlated, should be considered jointly in any outcome analysis.

Mean CPP has become an actively controlled variable and hence has lost its predictive power for outcome. It does not mean that traditional short term decreases in CPP ("CPP insults") have become any more benign, but they are probably better managed nowadays, and with baseline CPP above $65-70 \mathrm{~mm} \mathrm{Hg}$ they do not frequently produce ischaemia. This is, probably, one of the most spectacular "success stories" of CPP oriented protocols. However, there is still lack of "Class I" evidence indicating that CPP oriented therapy is clearly beneficial. Robertson et $\mathrm{l}^{36}$ compared CPP and ICP oriented therapies and showed a decrease in ischaemic insults in CPP oriented therapy but an increase in respiratory complications with no overall difference in outcome.

\section{ICP IN HYDROCEPHALUS AND BENIGN INTRACRANIAL HYPERTENSION}

ICP in chronic diseases should be interpreted slightly differently. The problems are related more to disturbance of the CSF circulation. Increased ICP signifies increased 

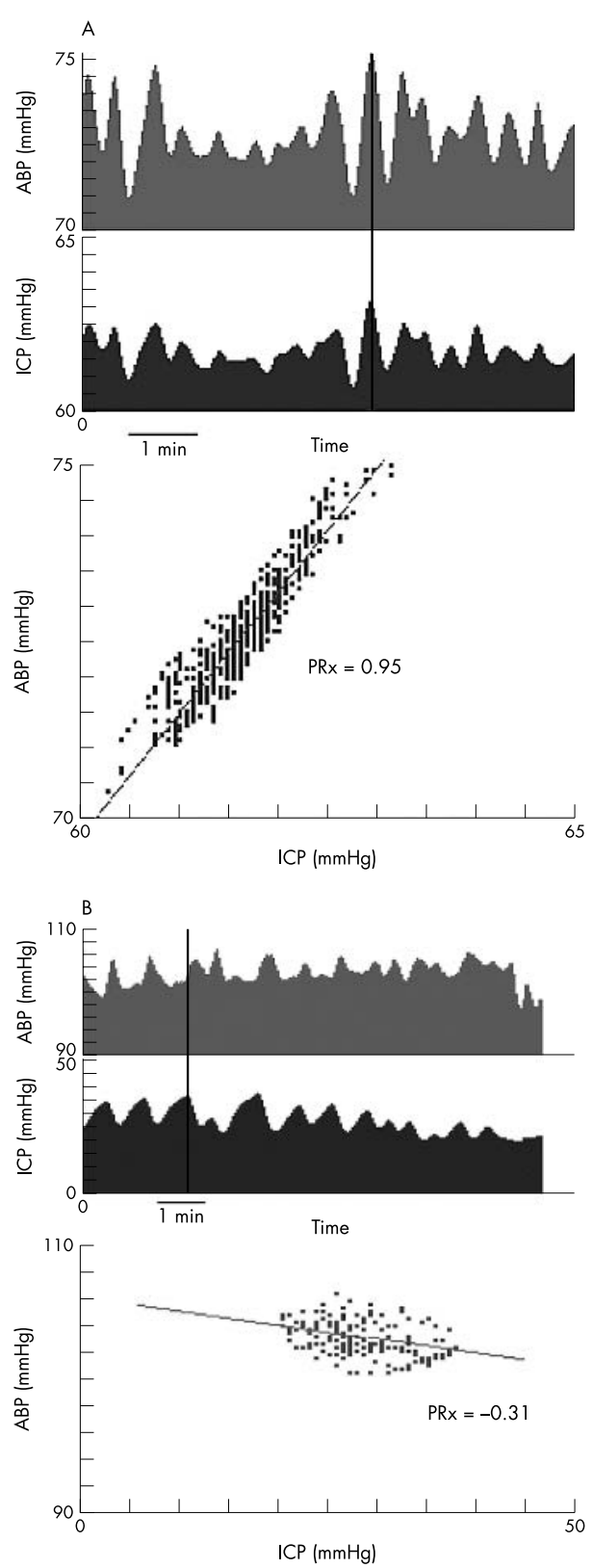

Figure 4 Relation between slow waves of arterial blood pressure (ABP) and intracranial pressure (ICP). (A) Slow waves in ICP and ABP produce obviously positive correlation (lower panel), giving a positive value of PRx. This indicated loss of cerebrovascular reserve. (B) Coherent waves both in ABP and ICP (upper panel) produced a negative correlation coefficient, when plotted on the regression graph (lower panel), giving clearly negative values of PRx. Taken from Czosnyka M, Smielewski P, Kirkpatrick $\mathrm{P}$, et al. Continuous assessment of the cerebral vasomotor reactivity in head injury. Neurosurgery 1997;41:11-1725 with permission from the publisher.

resistance to CSF outflow ${ }^{37}$ or increased cerebral venous outflow pressure, ${ }^{38}$ rather than exhausted cerebrospinal compensatory reserve created by a decreased volume in either one or both of the two main buffering componentsCSF and venous blood volume, as in head injury.

In more chronic conditions of ventricular dilatation, where ICP is not greatly raised, obstruction to CSF absorption may be confirmed by CSF infusion tests (ventricular or lumbar) taking care to adapt the technique to the site of any obstruction. ${ }^{39}$ The infusion study can be performed via the lumbar CSF space or via a preimplanted ventricular access

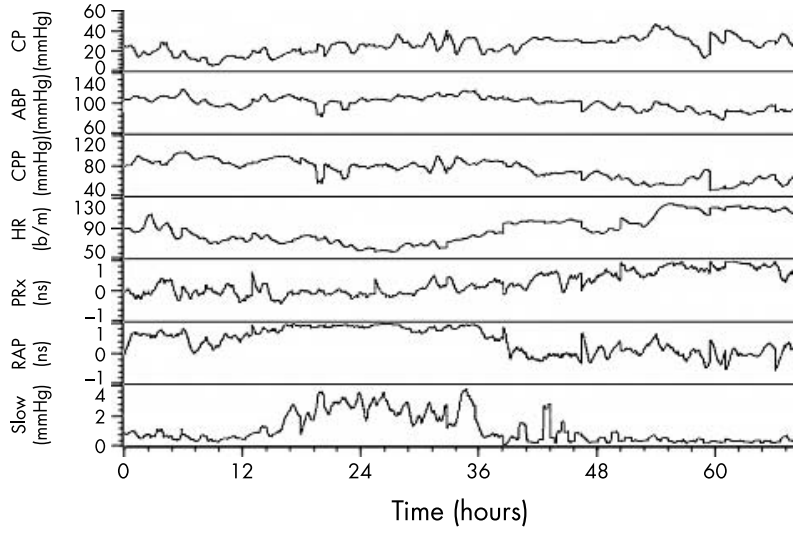

Figure 5 A typical example of intracranial hypertension in a patient who died. In this case the progressive increase in intracranial pressure (ICP) caused a severe fall in cerebral perfusion pressure (CPP) (time $48 \mathrm{~h}$ ), although normal values of arterial blood pressure (ABP) were maintained. The pressure-reactivity index (PRx), at first slightly above the normal range, started to climb in the second part of the monitoring (time $>40 \mathrm{~h}$ ) when the progressive increase in ICP was associated with CPP reduction; similarly RAP (index of compensatory reserve), initially above 0.6 , started to fall suggesting a loss in vasomotor reserve (time $>40 \mathrm{~h}$ ). Slow waves were low at the beginning, increased around 24 hours after injury and fell 36 hours after injury. Taken from Balestreri M, Czosnyka M. Acta Neurochir 2004; (in press) ${ }^{21}$ with permission from the publishers.

device. In both cases, two needles are inserted $(22 \mathrm{G}$ spinal needles for lumbar tests; $25 \mathrm{G}$ butterfly needles for ventricular studies). One needle is connected to a pressure transducer via a stiff saline-filled tube and the other to an infusion pump mounted on a purpose-built trolley containing a pressure amplifier and personal computer running software written inhouse. After 10 minutes of baseline measurement, the infusion of normal saline at a rate of $1.5 \mathrm{ml} / \mathrm{min}$ or $1 \mathrm{ml} /$ min (if the baseline pressure was higher than $15 \mathrm{~mm} \mathrm{Hg}$ ) is started and continued until a steady state ICP plateau is achieved (fig 6). If the ICP increases to $40 \mathrm{~mm} \mathrm{Hg}$, the infusion is interrupted. Following cessation of the saline infusion, ICP is recorded until it decreases to steady baseline levels. All compensatory parameters are calculated using computer supported methods based on physiological models of the CSF circulation. ${ }^{40}$ Baseline ICP and $\mathrm{R}_{\mathrm{CSF}}$ characterise the static properties of the CSF circulation. $\mathrm{R}_{\mathrm{CSF}}$ is calculated as the pressure increase during the infusion, divided by the infusion rate. A value below $13 \mathrm{~mm} \mathrm{Hg} /(\mathrm{ml} / \mathrm{min})$ characterises normal CSF circulation ${ }^{41}$; above $18 \mathrm{~mm} \mathrm{Hg} /(\mathrm{ml} / \mathrm{min})$ the CSF circulation is clearly disturbed ${ }^{42}$; between $13 \mathrm{~mm} \mathrm{Hg} /$ $(\mathrm{ml} / \mathrm{min})$ and $18 \mathrm{~mm} \mathrm{Hg} /(\mathrm{ml} / \mathrm{min})$ there is a grey zone, when other compensatory parameters and other clinical investigations should be considered to make a decision about shunting. Because the resistance to CSF outflow both in normal individuals ${ }^{43}$ and in patients with normal pressure hydrocephalus $(\mathrm{NPH})^{44}$ increases with age, it is very likely that the "critical threshold" of normal and abnormal $\mathrm{R}_{\mathrm{CSF}}$ should be also age adjusted.

The cerebrospinal elasticity coefficient (E1) and AMP waveform express the dynamic components of CSF pressure-volume compensation. ${ }^{45-47} \mathrm{El}$ describes the compliance of the CSF compartment according to the formula: compliance of CSF space $=C_{i}=1 /\left[\mathrm{El} \times\left(\mathrm{ICP}-\mathrm{p}_{0}\right)\right]$, where $\mathrm{p}_{0}$ is the unknown reference pressure level, representing the hydrostatic difference between the site of ICP measurement and the pressure indifferent point of the cerebrospinal axis. ${ }^{48}$ Cerebrospinal compliance is inversely proportional to ICP, therefore comparison between different subjects can be made only at the same level of the difference: $\mathrm{ICP}-\mathrm{p}_{0}$. El is 


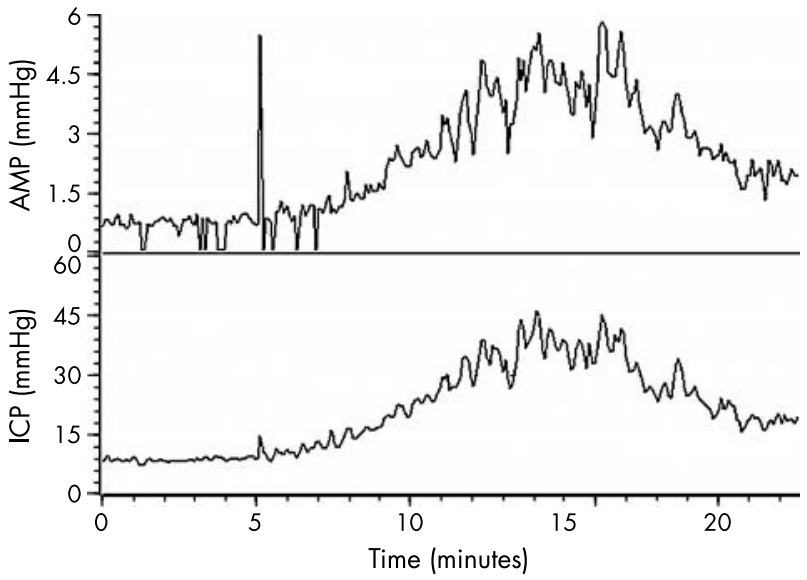

Figure 6 Infusion test. Pulse amplitude of intracranial pressure (ICP) (AMP) on upper graph and mean ICP (lower graph) plotted versus time. Normal saline infusion was started at 6 minutes of monitoring. ICP reached a level of $37 \mathrm{~mm} \mathrm{Hg}$. Infusion was discontinued at 17 minutes after start of recording. Resistance to CSF oufflow was elevated: $20 \mathrm{~mm}$ $\mathrm{Hg} /(\mathrm{ml} / \mathrm{min})$. AMP always increases proportionally to an increase of mean ICP.

independent of ICP, thus this coefficient is a much more convenient parameter when comparing individual patients. A low value of El (less than $0.2 / \mathrm{ml}$ ) is specific for a compliant system, whereas a higher value indicates decreased pressurevolume compensatory reserve.

The AMP increases proportionally when the mean ICP rises. The proportionality ratio (the AMP/P index) characterises both the elastance of the cerebrospinal space and the transmission of arterial pulsations to the CSF compartment. ${ }^{41}$ Finally, the production of CSF fluid can be estimated using Davson's equation. However, the sagittal sinus pressure $\left(\mathrm{P}_{\mathrm{SS}}\right)$ is unknown and cannot be easily measured without increasing the invasiveness of the whole procedure. Consequently, the $\mathrm{P}_{\mathrm{SS}}$ and CSF formation are estimated jointly using a nonlinear model using the least square distance method during the computerised infusion test. ${ }^{40}$ It is important to mention that such an "estimate" of CSF production rate approximates CSF absorption, rather than the actual production rate. It is based upon the assumption that all circulating CSF is reabsorbed via the arachnoid granulations. In cases where significant CSF suffusion into brain parenchyma occurs, CSF production may be grossly underestimated.

An infusion study may be useful for the assessment of shunt function in vivo. The end-equilibrium pressure achieved during the test should not significantly exceed the shunt's operating pressure increased by the hydrodynamic resistance of the opened shunt multiplied by the infusion rate. These values are readily available from the UK shunt evaluation laboratory (Academic Neurosurgical Unit, Addenbrooke's Hospital, Cambridge, UK) and can be used in clinical measurements to confirm shunt malfunction objectively. ${ }^{49}$ This is particularly important as an increasing number of shunt revisions obviously impedes the chance for uneventful management of hydrocephalus. ${ }^{50}$

Overnight ICP monitoring in patients with NHP may reveal a high incidence of slow waves during sleep which is a very helpful prognostic sign for the outcome following shunting $^{51}{ }^{52}$ (fig 7A). Benign intracranial hypertension seldom requires more than CSF pressure monitoring through a lumbar catheter or needle for an hour. When overnight ICP monitoring is performed in a patient with benign intracranial hypertension, baseline ICP is usually increased, the amplitude and frequency of "slow waves" is moderate, but the increased RAP index indicates reduced cerebrospinal
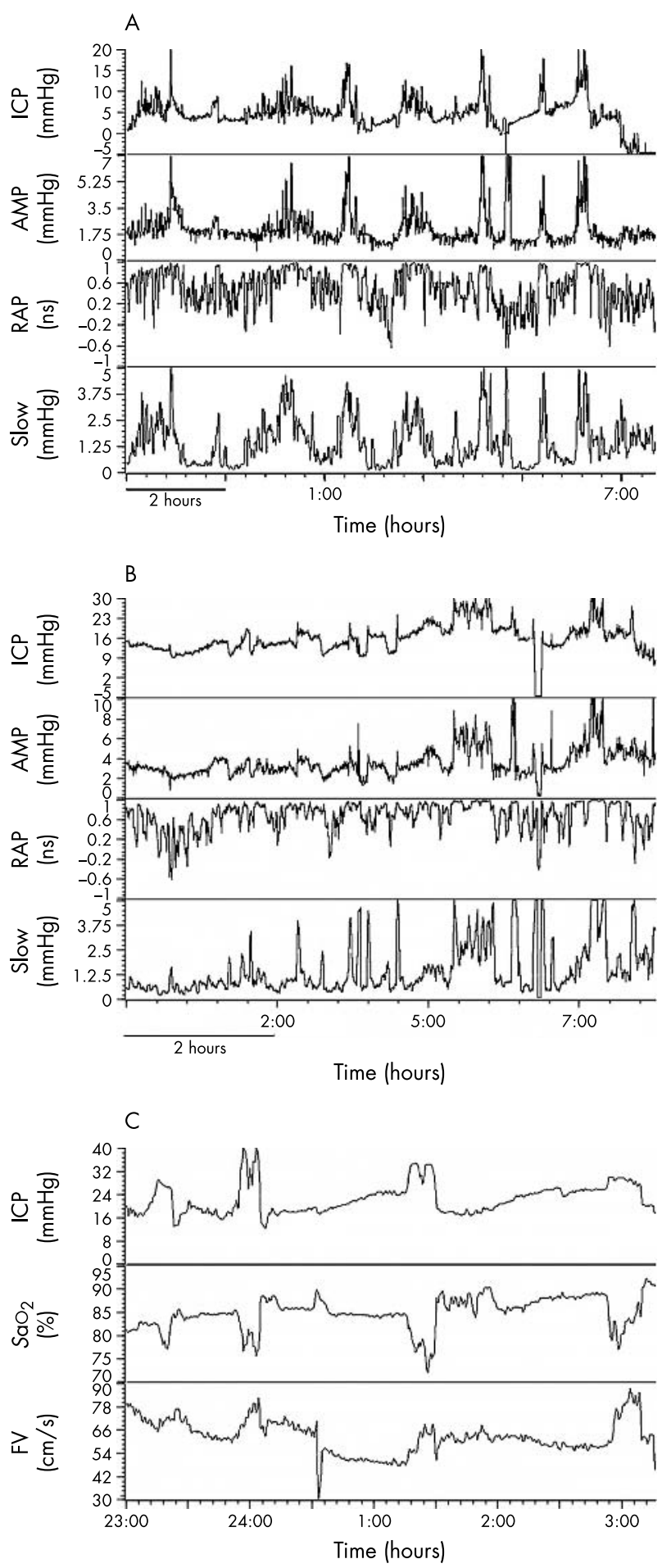

Figure 7 Overnight monitoring of intracranial pressure. (A) Normal pressure hydrocephalus: baseline pressure normal $(5 \mathrm{~mm} \mathrm{Hg})$ with periodical vasogenic increases reaching $20 \mathrm{~mm} \mathrm{Hg}$ (ever hour), with associated decrease of compensatory reserve (RAP increasing towards +1 ) and an increase in magnitude of slow waves. These are vasogenic events, most frequently triggered by the REM phase of sleep. (B) Benign intracranial hypertension: baseline ICP elevated to $20 \mathrm{~mm} \mathrm{Hg}$ with limited dynamics (although vasogenic waves clearly present around 5$6 \mathrm{am}$ ) and with permanently reduced compensatory reserve (RAP close to +1$)$. (C) Intermittent hypertension due to sleep apnoea-case reported in reference 53. ICP, mean ICP level (1 minute averaged); AMP, pulse amplitude of ICP; RAP, index of compensatory reserve; Slow, magnitude of slow waves of ICP; $\mathrm{FV}$, blood flow velocity; $\mathrm{SaO}_{2}$, arterial blood saturation, REM, rapid eye movement. 
compensatory reserve (fig 7B). Intermittent intracranial hypertension caused by sleep apnoea syndrome may cause symptoms similar to benign intracranial hypertension. Multimodal monitoring including ICP, Doppler blood flow velocity, and arterial blood oxygen saturation is useful (fig 7C). ${ }^{53}$

Although a number of centres employ ICP measurement and infusion studies, the selection of patients has not been shown to be more precise than when using the CSF tap test. ${ }^{54}$ Positive predictive power of the increased resistance to CSF outflow is generally high, but the negative predictive power is low-that is, patients with normal resistance to CSF outflow may sometimes improve following shunting. However, the same problem concerns CSF tap tests. ${ }^{55}$ Extending the period of CSF drainage to 72 hours seems to be an efficient solution but increases hospital stay and risk of complications.

\section{ATTEMPTS TO MEASURE ICP AND CPP NON- INVASIVELY}

It would be very helpful to measure ICP or CPP without invasive transducers. Transcranial Doppler examination, ${ }^{16}$ tympanic membrane displacement, ${ }^{15}$ and ultrasound "time of flight" techniques have been advocated. ${ }^{17}$

The description of transcranial Doppler sonography by Aaslid et al in 1982 permitted bedside monitoring of one index of cerebral blood flow, non-invasively, repeatedly, and even continuously. ${ }^{56}$ The problem has been that it is a "big tube technique", which measures flow velocity in branches of the circle of Willis, most commonly the middle cerebral artery (MCA). Compliant branches of the MCA can be compared with two physiological pressure transducers. The pattern of blood flow within these tubes is certainly modulated by transmural pressure-that is, CPP, and the distal vascular resistance (also modulated by the $\mathrm{CPP}$ ). But what is the calibration factor and how should we compensate for unknown non-linear distortion?

There is a reasonable correlation between the pulsatility index of MCA velocity and CPP after head injury but absolute measurements of CPP cannot be extrapolated. ${ }^{57}$ Others have suggested that "critical closing pressure" derived from flow velocity and arterial pressure waveform approximated the ICP. ${ }^{58}$ The accuracy of this method has, however, never been satisfactory. ${ }^{59}$

Aaslid et $a l^{60}$ suggested that an index of CPP could be derived from the ratio of the amplitudes of the first harmonics of the ABP and the MCA velocity (detected by transcranial Doppler sonography) multiplied by mean flow velocity. Recently, a method for the non-invasive assessment of CPP has been reported, derived from mean arterial pressure multiplied by the ratio of diastolic to mean flow velocity.$^{61}{ }^{62}$ This estimator can predict real CPP with an error of less than $10 \mathrm{~mm} \mathrm{Hg}$ for more than $80 \%$ of measurements. This is of potential benefit for the continuous monitoring of changes in real CPP over time in situations where the direct measurement of CPP is not readily available.

A more complex method aimed at the non-invasive assessment of ICP has been introduced and tested by Schmidt et al. ${ }^{18}$ The method is based on the presumed linear transformation between arterial pressure and ICP waveforms. Coefficients of this transformation are derived from the database of real ABP and ICP recordings. Similar linear transformation is built, using the same database between flow velocity and arterial pressure. Then the model assumes linear relationship between arterial pressure and flow velocity and arterial pressure to ICP transformations. Multiple regression coefficients are calculated. Finally, for each prospective study, ICP is calculated using $\mathrm{ABP}$ to ICP transformation, formed from $\mathrm{ABP}$ to flow velocity transformation transposed using precalculated regression coefficients.

\section{IS ICP MONITORING USEFUL}

The continuous measurement of ICP is an essential modality in most brain monitoring systems. After a decade of enthusiastic attempts to introduce new modalities for brain monitoring (tissue oxygenation, microdialysis, cortical blood flow, transcranial Doppler ultrasonography, jugular bulb oxygen saturation) it is increasingly obvious that ICP is robust, only moderately invasive, and can be realistically conducted in regional hospitals.

Although there has been no randomised controlled trial about influence of ICP monitoring on overall outcome after following head injury, recent audit ${ }^{63}$ shows almost twofold lower mortality in neurosurgical centres, where ICP is usually monitored, versus general intensive care units, where it is not monitored. However, the availability of ICP monitoring is not the only difference between neurosurgical and general intensive care units that might explain the difference in mortality after head injury.

ICP waveform contains valuable information about the nature of cerebrospinal pathophysiology. Autoregulation of cerebral blood flow and compliance of cerebrospinal system are both expressed in ICP. Methods of waveform analysis are useful both to derive this information and to guide the management of patients.

The value of ICP in acute states such as head injury, poor grade subarachnoid haemorrhage, and intracerebral haematoma depends on a close link between monitoring and therapy. CPP oriented protocols, ${ }^{6465}$ osmotherapy $^{66}$ and the "Lund protocol" cannot be conducted correctly without ICP guidance. A decision about decompressive craniectomy should be supported by the close inspection of the trend of ICP and, preferably, by information derived from its waveform. ${ }^{67}$ In encephalitis, ${ }^{68}$ acute liver failure, ${ }^{69}$ and cerebral infarction after stroke, ${ }^{70}$ ICP monitoring is used less commonly, however, an increasing number of reports highlight its importance.

A slightly different methodology for CSF pressure interpretation is applied in chronic states such as hydrocephalus or benign intracranial hypertension. In the first case assessment of CSF pressure-volume compensation and circulation are essential to optimise patient management. ${ }^{42} 71$ Volume-adding tests with parallel measurement of ICP and/ or overnight ICP monitoring with waveform analysis have a special role. In patients with a shunt in situ, who present with persistent or recurring clinical symptoms, it helps to avoid unnecessary shunt revisions. This is particularly important as patients with a history of multiple shunt revision have a lower chance to achieve good outcome in the future. In benign intracranial hypertension ${ }^{72}$ or craniostenosis $^{73}$ ICP monitoring has been documented as useful both for diagnosis and to document response to therapy.

In summary, ICP is a complex modality, which contains combined information about cerebral compensatory and CBF regulation mechanisms. Control of ICP requires continuous monitoring. ICP-derived indices help to understand the pathophysiology of developing events and facilitate patient care.

\section{ACKNOWLEDGEMENTS}

Many thanks to our colleagues participating in the head injury monitoring programme in Cambridge, UK, 1991-2002, whose experience, knowledge, and clinical material helped me to write this review: P Al-Rawi, D Chattfield, C Turner, M Balestreri, M Hiler, B Owler, A Pena, P Smielewski, SK Piechnik, LA Steiner, E Guazzo, PC Whetfield, P Minhas, M Soehle, R Kett-White, P Hutchinson, E Schmidt, B Schmidt, A Kumar, A Raabe, M Schuhmann, PJ Kirkpatrick, FK Matta, A Gupta, DK Menon, and nursing and 
research staff from Neuro Critical Care Unit and Wolfson Brain Imaging Centre.

\author{
Authors' affiliations \\ M Czosnyka*, J D Pickard, Academic Neurosurgical Unit, University of \\ Cambridge Clinical School, Cambridge, UK
}

*M Czosnyka is on leave from Warsaw University of Technology, Poland.

Grant support: Medical Research Council No G9439390 ID 65883.

Competing interests: none declared

This review is complementary to a review of raised ICP published in Hughes RAC, ed. Neurological Emergencies. London: BMJ Books, 2003.

\section{REFERENCES}

1 Marmarou A, Maset AL, Ward JD, et al. Contribution of CSF and vascular factors to elevation of ICP in severely head-injured patients. J Neurosurg 1987;66:883-90

2 Czosnyka M, Richards HK, Czosnyka Z, et al. Vascular components of cerebrospinal fluid compensation. J Neurosurg 1999;90:752-9.

3 Davson H, Hollingsworth G, Segal MB. The mechanism of drainage of the cerebrospinal fluid. Brain 1970;93:665-78.

4 Miller JD, Stanek A, Langfitt TW. Concepts of cerebral perfusion pressure and vascular compression during intracranial hypertension. Prog Brain Res 1972;35:411-32.

5 Steiner LA, Czosnyka M, Piechnik SK, et al. Continuous monitoring of cerebrovascular pressure reactivity allows determination of optimal cerebral perfusion pressure in patients with traumatic brain injury. Crit Care Med 2002;30:733-8.

6 Asgeirsson B, Grande PO, Nordstrom CH. A new therapy of post-trauma brain oedema based on haemodynamic principles for brain volume regulation. Intensive Care Med 1994;20:260-7.

7 Guillaume J, Janny P. Manometrie intracranienne continué interest de la methode et premiers resultants. Rev Neurol (Paris) 1951;84:131-42.

8 Lundberg N. Continuous recording and control of ventricular fluid pressure in neurosurgical practice. Acta Psychiatr Neurol Scand 1960;36/suppl 149): 1-193.

9 Ghajar J. Intracranial pressure monitoring techniques. New Horizions 1995;3:395-9

10 Czosnyka M, Czosnyka Z, Pickard JD. Laboratory testing of three intracranial pressure microtransducers: Technical report. Neurosurgery 1996;38:219-24.

11 Wolfla CE, Luerssen TG, Bowman RM, et al. Brain tissue pressure gradients created by expanding frontal epidural mass lesion. J Neurosurg 1996:84:642-7.

12 Piper I, Barnes A, Smith D, et al. The Camino intracranial pressure sensor: is it optimal technology? An internal audit with a review of current intracranial pressure monitoring technologies. Neurosurgery 2001;49:1158-64.

13 Chambers IR, Siddique MS, Banister K, et al. Clinical comparison of the Spiegelberg parenchymal transducer and ventricular fluid pressure. I Neurol Neurosurg Psychiatry 2001;71:383-5.

14 Owler BK, Fong KC, Czosnyka Z. Importance of ICP monitoring in the investigation of CSF circulation disorders. Br J Neurosurg 2001;15:439-40.

15 Reid A, Marchbanks RJ, Martin R, et al. Mean intracranial pressure monitoring by an audiological technique-a pilot study. I Neurol Neurosurg Psychiatry 1989;52:610-12.

16 Schoser BG, Riemenschneider N, Hansen HC. The impact of raised intracranial pressure on cerebral venous hemodynamics: a prospective venous transcranial Doppler ultrasonography study. J Neurosurg 1999;91:744-9.

17 Petkus V, Ragauskas A, Jurkonis R. Investigation of intracranial media ultrasonic monitoring model. Ultrasonics 2002;40:829-33.

18 Schmidt B, Klingelhofer J, Schwarze JJ, et al. Noninvasive prediction of intracranial pressure curves using transcranial Doppler ultrasonography and blood pressure curves. Stroke 1997;28:2465-72.

19 Albeck MJ, Borgesen SE, Gjerris F, et al. Intracranial pressure and cerebrospinal fluid oufflow conductance in healthy subjects. J Neurosurg 1991;74:597-600.

20 Chapman PH, Cosman ER, Arnold MA. The relationship between ventricular fluid pressure and body position in normal subjects and subjects with shunts: a telemetric study. Neurosurgery 1990;26:181-9.

21 Balestreri M, Czosnyka M. Intracranial hypertension: what additional information can be derived form ICP waveform after head injury? Acta Neurochir, 2004; (in press)..

22 Lofgren J, von Essen C, Zwetnow NN. The pressure-volume curve of the cerebrospinal fluid space in dogs. Acta Neurol Scand 1973:49:557-74.

23 23. Avezaat CJ, van Eijndhoven JH, Wyper DJ. Cerebrospinal fluid pulse pressure and intracranial volume-pressure relationships. J Neurol Neurosurg Psychiatry 1979;42:687-700.

24 Muizelaar JP, Ward JD, Marmarou A, et al. Cerebral blood flow and metabolism in severely head-injured children. Autoregulation. Neurosurg 1989;71:72-6

25 Czosnyka M, Smielewski P, Kirkpatrick P, et al. Continuous assessment of the cerebral vasomotor reactivity in head injury. Neurosurgery 1997:41:11-17.

26 Miller JD, Pickard JD. Intracranial volume pressure studies in patients with head injury. Injury 1974;5:265-8.
27 Piper IR, Miller JD, Whittle IR, et al. Automated time-averaged analysis of craniospinal compliance (short pulse response). Acta Neurochir Suppl (Wien) 1990;51:387-90.

28 Piper I, Spiegelberg A, Whittle I, et al. A comparative study of the Spiegelberg compliance device with a manual volume-injection method: a clinical evaluation in patients with hydrocephalus. Br J Neurosurg 1999;13:581-6.

29 Yau YH, Piper IR, Contant CF, et al. Clinical experience in the use of the Spiegelberg automated compliance device in the assessment of patients with hydrocephalus. Acta Neurochir Suppl 2002;81:171-2.

30 Robertson C, Narayan R, Contant C, et al. Clinical experience with continuous monitoring of intracranial compliance. J Neurosurg 1989;71:673-80.

31 Portnoy HD, Chopp M, Branch C, et al. Cerebrospinal fluid pulse waveform as an indicator of cerebral autoregulation. J Neurosurg 1982;56:666-78.

32 Takizawa H, Gabra-Sanders T, Miller JD. Changes in the cerebrospinal fluid pulse wave spectrum associated with raised intracranial pressure. Neurosurgery 1987;20:355-61.

33 Piper I, Miller JD, Dearden $M$, et al. System analysis of cerebrovascular pressure transmission: an observational study in head injured patients. J Neurosurg 1990;73:871-80.

34 Foltz EL, Blanks JP, Yonemura K. CSF pulsatility in hydrocephalus: respiratory effect on pulse wave slope as an indicator of intracranial compliance. Neurol Res 1990;12:67-74.

35 Lemaire JJ, Khalil T, Cervenansky F, et al. Slow pressure waves in the cranial enclosure. Acta Neurochir (Wien) 2002;144:243-54.

36 Robertson CS, Valadka AB, Hannay HJ, et al. Prevention of secondary ischemic insults after severe head injury. Crit Care Med 1999;27:2086-95.

37 Borgesen SE, Gjerris F. Relationships between intracranial pressure, ventricular size, and resistance to CSF oufflow. J Neurosurg 1987;67:535-9.

38 Higgins JN, Owler BK, Cousins $C$, et al. Venous sinus stenting for refractory benign intracranial hypertension. Lancet 2002;359:228-30.

39 Katzman R, Hussey F. A simple constant-infusion manometric test for measurement of CSF absorption. I Rationale and method. Neurology 1970;20:534-44.

40 Czosnyka M, Whitehouse $H$, Smielewski $P$, et al. Testing of cerebrospinal compensatory reserve in shunted and non-shunted patients: a guide to interpretation based on observational study. J Neurol Neurosurg Psychiatry 1996;60:549-58.

41 Borgesen SE, Gjerris F. The predictive value of conductance to outflow of CSF in normal pressure hydrocephalus. Brain 1982;105:65-86.

42 Boon AJ, Tans JT, Delwel EJ, et al. Dutch normal-pressure hydrocephalus study: prediction of outcome after shunting by resistance to outflow of cerebrospinal fluid. J Neurosurg 1997:87:687-93.

43 Albeck MJ, Skak C, Nielsen PR, et al. Age dependency of resistance to cerebrospinal fluid oufflow. J Neurosurg 1998;89:275-8.

44 Czosnyka M, Czosnyka ZH, Whiffield PC, et al. Age dependence of cerebrospinal pressure-volume compensation in patients with hydrocephalus. J Neurosurg 2001;94:482-6.

45 Avezaat CJJ, Eijndhoven JHM. Cerebrospinal fluid pulse pressure and craniospinal dynamics. A theoretical, clinical and experimental study [Thesis] The Hague: Jongbloed A, 1984

46 Ekstedt J. CSF hydrodynamic studies in man. Method of constant pressure CSF infusion. J Neurol Neurosurg Psychiatry 1977;40:105-19.

47 Marmarou A, Shulman K, Rosende RM. A non-linear analysis of CSF system and intracranial pressure dynamics. J Neurosurg 1978;48:332-44.

48 Raabe A, Czosnyka M, Piper I, et al. Monitoring of intracranial compliance: correction for a change in body position. Acta Neurochir (Wien) 1999;141:31-6.

49 Czosnyka ZH, Czosnyka M, Pickard JD. Shunt testing in-vivo: a method based on the data from the UK shunt evaluation laboratory. Acta Neurochir Suppl 2002;81:27-30.

50 Tuli S, Drake J, Lawless J, et al. Risk factors for repeated cerebrospinal shunt failures in pediatric patients with hydrocephalus. J Neurosurg 2000;92:31-8.

51 Pickard JD, Teasdale G, Matheson M, et al. Intraventricular pressure waves the best predictive test for shunting in normal pressure hydrocephalus. In: Shulman K, Marmarou A, Miller JD, Becker DP, Hochwald GM, Brock M, eds. Intracranial Pressure IV. Berlin: Springer-Verlag, 1980:498-500.

52 Symon L, Dorsch NWC, Stephens RJ. Pressure waves in so-called lowpressure hydrocephalus. Lancet 1972:2:1291-2.

53 Kirkpatrick PJ, Meyer T, Sarkies N, et al. Papilloedema and visual failure in a patient with nocturnal hypoventilation. J Neurol Neurosurg Psychiatry 1994:57:1546-7.

54 Kahlon B, Sundbarg G, Rehncrona S. Comparison between the lumbar infusion and CSF tap tests to predict outcome after shunt surgery in suspected normal pressure hydrocephalus. J Neurol Neurosurg Psychiatry 2002;73:721-6.

55 Walchenbach R, Geiger E, Thomeer RT, et al. The value of temporary external lumbar CSF drainage in predicting the outcome of shunting on normal pressure hydrocephalus. J Neurol Neurosurg Psychiatry 2002;72:503-6.

56 Aaslid R, Markwalder TM, Nornes H. Noninvasive transcranial Doppler ultrasound recording of flow velocity in basal cerebral arteries. J Neurosurg 1982:57:769-74.

57 Chan KH, Miller JD, Dearden NM, et al. The effect of changes in cerebral perfusion pressure upon middle cerebral artery blood flow velocity and jugular bulb venous oxygen saturation after severe brain injury. J Neurosurg 1992;77:55-61.

58 Dewey RC, Pieper HP, Hunt WE. Experimental cerebral hemodynamics. Vasomotor tone, critical closing pressure, and vascular bed resistance. Neurosurgery 1974:41:597-606.

59 Czosnyka M, Smielewski P, Piechnik S, et al. Critical closing pressure in cerebrovascular circulation. J Neurol Neurosurg Psychiatry 1999;66:606-11. 
60 Aaslid R, Lundar T, Lindegaard K-F, et al. Estimation of cerebral perfusion pressure from arterial blood pressure and transcranial Doppler recordings. In: Miller JD, Teasdale GM, Rowan JO, Galbraith SL, Mendelow AD, eds. Intracranial Pressure VI. Berlin: Springer-Verlag, 1986;229-31).

61 Czosnyka M, Matta BF, Smielewski P, et al. Cerebral perfusion pressure in head-injured patients: a noninvasive assessment using transcranial Doppler ultrasonography. J Neurosurg 1998;88:802-8.

62 Schmidt EA, Czosnyka M, Matta BF, et al. Non-invasive cerebral perfusion pressure (nCPP): evaluation of the monitoring methodology in head injured patients. Acta Neurochir Suppl 2000;76:451-2.

63 Patel HC, Bouamra O, King A, et al. Mortality after head trauma. Effect of neurosurgical care. Euroacademia Multidisciplinaria Neurotraumatologica. Graz, 2003. [Abstract] Acra Neurochir (Wein) 2003;145:1135-48.

64 Rosner MJ, Rosner SD, Johnson AH. Cerebral perfusion pressure: Management protocol and clinical results. J Neurosurg 1995;83:949-62.

65 Patel HC, Menon DK, Tebbs S, et al. Specialist neurocritical care and outcome from head injury. Intensive Care Med 2002;28:547-53.

66 Bullock R. Mannitol and other diuretics in severe neurotrauma. New Horizons 1995;3:448-52.
67 Whiffield PC, Patel $\mathrm{H}$, Hutchinson PJ, et al. Bifrontal decompressive craniectomy in the management of posttraumatic intracranial hypertension. Br J Neurosurg 2001; 15:500-7.

68 Rebaud P, Berthier JC, Hartemann E, et al. Intracranial pressure in childhood central nervous system infections. Intensive Care Med 1988;14:522-5.

69 Keays RT, Alexander GJ, Williams R. The safety and value of extradural intracranial pressure monitors in fulminant hepatic failure. $J$ Hepato 1993;18:205-9.

70 Schwab S, Aschoff A, Spranger $M$, et al. The value of intracranial pressure monitoring in acute hemispheric stroke. Neurology 1996;47:393-8.

71 Tisell $M$, Edsbagge $M$, Stephensen $H$, et al. Elastance correlates with outcome after endoscopic third ventriculostomy in adults with hydrocephalus caused by primary aqueductal stenosis. Neurosurgery 2002;50:70-7.

72 Sussman JD, Sarkies N, Pickard JD. Benign intracranial hypertension. Pseudotumour cerebri: idiopathic intracranial hypertension. Adv Tech Stand Neurosurg 1998;24:261-305.

73 Tuite GF, Evanson J, Chong WK, et al. The beaten copper cranium: a correlation between intracranial pressure, cranial radiographs, and computed tomographic scans in children with craniosynostosis. Neurosurgery 1996;39:691-9. 